\title{
Design of Intelligent Power Metering System with Pre-pay Based on ARM Cortex-M3
}

\author{
Xie-Qiu ZHAO' ${ }^{1}$, Yan-Ming CHENG ${ }^{2 *}$, Shan-Jie TANG ${ }^{2}$ and Lan-Jing GONG \\ ${ }^{1}$ College of Computer Science \& Technology Engineering, Beihua University, China \\ ${ }^{2}$ College of Electrical \& Information Engineering, Beihua University, China \\ *Corresponding author
}

Keywords: Pre-pay, Intelligent Metering, ARM, SPI.

\begin{abstract}
This paper presents one pre-pay intelligent power metering management system based on ARM Cortex-M3 which is composed of power supply control cabinet and PC server, between them data transmission is carried out through TCP/IP. The ADE7753AR single energy chip with SPI communication interface is used to design the energy metering module. The embedded ARM processor and STM32F103ZE chip with Cortex-M3 kernel are used as the core of the accounting management controller. The control cabinet is connected to the metering module and the controller through the RS-485 bus, which can achieve higher reliability and the performance price ratio of the system. The centralized management mode is adopted to implement mobile phone campus card pay, which will provide convenient and quick, and has broad application prospect.
\end{abstract}

\section{Introduction}

Energy meter is an important component of power energy management, and its performance will directly affect the efficiency and level of power energy management [1]. With the commercial transformation of the mode of production and management mode of China's electric power industry, the electricity as a commodity into the market, urgent need to establish an accurate, scientific and reasonable modern electric energy metering and management system puts forward increasing higher requirements on the performance and structure of power energy meter. The development of modern electronic technology, computer technology, communication technology and network technology in modern times provides technical support for the improvement and optimization of energy meter.

This paper designs the ARM based prepaid intelligent power metering management system is to follow the [2] standard of power system and secondarily develop power metering management system with the characteristics of strong professional, high degree of automation, easy to use, high performance and high reliability in college student apartments and residential apartments. The system is composed of power supply control cabinet and PC server. It has the functions of centralized power collection, power management and control. Intelligent power management system with power centralized measurement, schedule control, electricity time overload control, electric load limit, electricity network data real-time query, user management, room allocation, deposit records, cost statistics, real-time monitoring, illegal electricity records management functions and other functions, can automatically read the electricity, settlement, archive, and to warn the customer arrearage power, power control, which saves a lot of manual meter reading time.

\section{System Scheme Design}

The system scheme is depicted in Fig.1, which is composed of power supply control cabinet and PC server. The control cabinet is composed of an electric energy metering module, a query manager, a metering controller and an air circuit breaker. 
A server can control multiple power supply control cabinets. The server collects and stores the power supply control cabinet data and sends the control instruction, and the server computer and the power supply control cabinet data backup each other. The power supply control cabinet can work independently even after the computer fails or shuts down, and can complete the energy metering of each shunt, and automatically store the relevant data, and has the power off protection function [3].

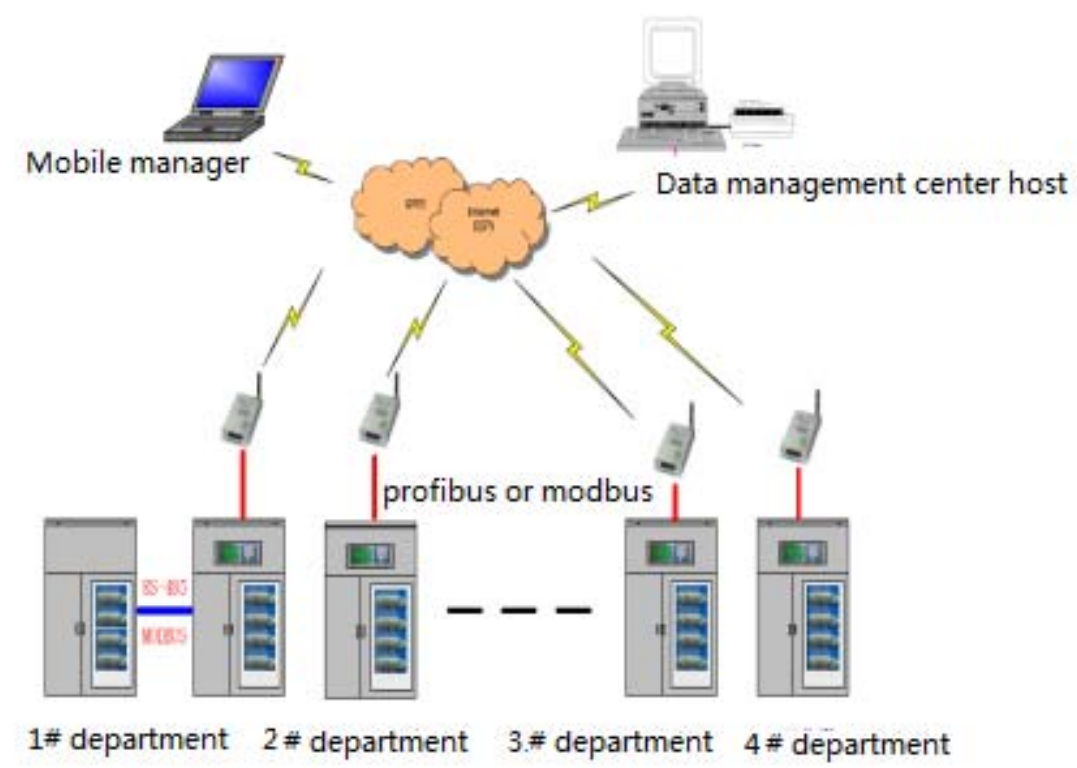

Fig.1 System diagram

\section{Hardware part design}

\subsection{Design of electric energy metering module}

The ADE7753AR energy measuring chip with SPI communication interface is designed, which can realize high-precision measurement of electric energy and digital calibration compensation. It is convenient for users to set up and calibrate operations. By the voltage sampling resistor divider, using $50 \mathrm{~A} / 20 \mathrm{~mA}$ micro current transformer to obtain current sampling with pulse constant of 3200, by SPI bus, metering controller set up the ADE7753 and read the information to complete the energy accumulation and energy costs, and which can complete electricity consumption record of 60 days [4]. Due to the metering controller is provided with independent storage and RTC circuits, the utility model can work independently when the inquiry manager or other metering controllers fail, without affecting the metering and accounting functions. Comparing to the previous pure analog measurement chip and mechanical meter, the application of ADE7753AR reduces system cost and improves the reliability, which meets the demand of the power design of real-time, high precision and low power consumption system. The function diagram of the metering controller is shown in Fig. 2. 


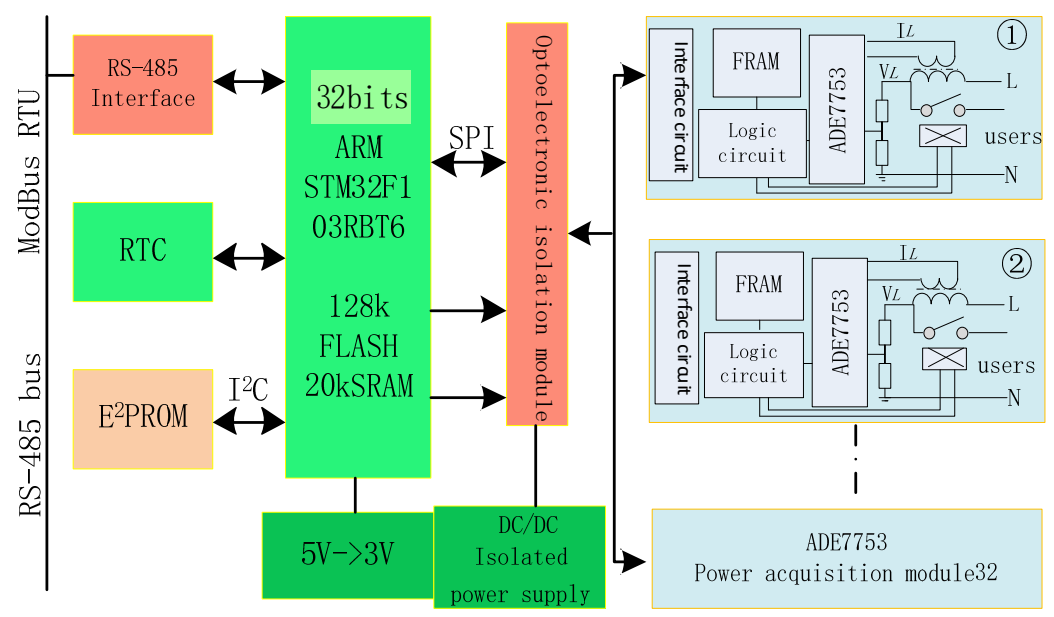

Fig.2 Function diagram of metering controller and metering module

\subsection{Design of metering control unit}

Metering controller uses 32 bits STM32F103RBT6 embedded processor with Cortex-M3 kernel implementation, the processor has FLASH memory chip $128 \mathrm{~K}$, it can be configured for program memory or data storage. The SRAM memory with $20 \mathrm{~K}$ in the chip can meet the requirements of data calculation and communication. The main frequency of $72 \mathrm{MHz}$ can easily meet the technical requirements of high speed power acquisition system. The serial JTAG and SWD interface provide the online simulation function, which reduces the difficulty of hardware design and debugging, and has high performance price ratio.

Through the SPI bus with hcpl0601 high speed photoelectric isolation interface, each STAM32F103RBT6 processor samples data of up to 32-way power energy metering module, and processes these data; at the same time, electric power and current detection circuit, the dynamic parameters of the circuit load characteristics are processed by the automatic identification module to complete the malicious load detection and overload, over-current protection function.

The metering controller consists of RTC module and RS-485 communication interface. The query manager collects the measurement parameters of each metering controller through the Modbus RTU protocol[5], sets the user control parameters and the cost, and synchronously RTC the clock. According to the set parameters, the controller can complete the collection of user electrical parameters, the calculation of the cost, the setting of malicious loads and the timing switch.

\section{Software Design}

\subsection{Metering Controller Design}

The metering controller software is designed by using $\mathrm{C}$ programming language modular design. The program function module adopts data stream driving mode, and the module decides the output state according to the result of static data and dynamic data. Fig. 3 is the flow of data between modules. 


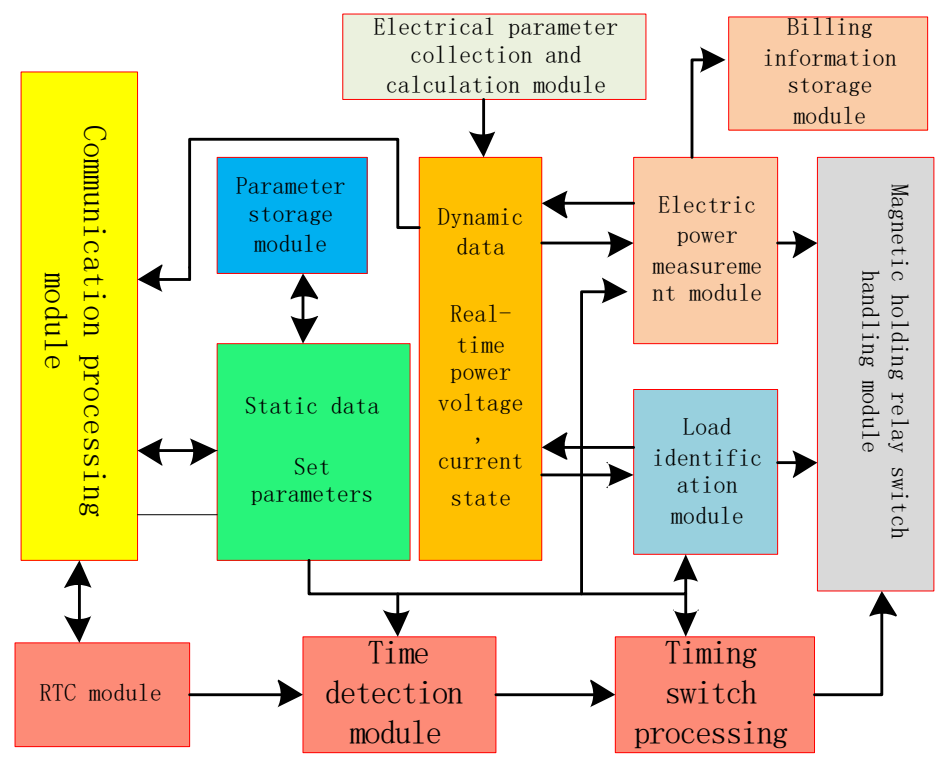

Fig.3 Data flow drive of function module

\subsection{Query manager design}

The query manager realizes the real-time electric energy data of each metering controller to be displayed and processed, and responds to the commands and parameters set by the PC through the TCP/IP protocol. The query manager uses 32-bit embedded processor STM32F103RCT6, with RS-485 bus and 10/100MHz LAN interface, which provides high-speed communication capability with metering controller and PC. Each manager has a real-time running clock, and can be synchronized by the PC, so as to ensure the unity of time control. At the same time, the query manager extends the $4 * 8$ keyboard and $240 * 320$ pixel displayer communication interface to realize the inquiry, setting and payment function of the power energy.

\subsection{Communication module design}

The system uses RS-485 and LAN two communication interface with the industry standard Modbus RTU and TCP/IP communication protocol to connect meter, query manager and PC server, which can achieve online parameter setting and query function[3]. The system may also adopt other kinds of communication methods, such as wireless data transmission module, GPRS or WLAN, etc. the design of various communication modes makes the system networking more flexible and convenient, and the market competition is stronger.

\subsection{Software development environment}

The measurement data and the query manager are programmed with $\mathrm{C}$ language in $\mathrm{uVision} 5 \mathrm{IDE}$ platform of the German company Keil, by using RTX real-time operating system makes modules and code more convenient, easily to maintain the system. PC management software is implemented by Labview2014 graphical programming. It can easily realize communication processing, data display and storage, and the report and curve query can be directly invoked by the module. The background database uses SQL database management engine to complete the storage, query and report of power data.

\section{Conclusions}

In this paper, based on the design of prepaid ARM intelligent power energy management system has electric power metering, time control, load control, real-time monitoring and other functions, to facilitate centralized meter and management, and prevent all kinds of leakage, the overload of electricity stealing phenomenon. The system adopts centralized management mode and implements 
mobile phone campus card pay. It is convenient and quick to use and has high reliability and cost performance.

\section{Acknowledgments}

This work was supported by the Scientific and Technological Planning Project of Beihua University, China (No. YB201620).

\section{References}

[1] Liu Xiaozhao, Chen Zhifeng. Design of student dormitory power management system based on Zigbee technology [J]. Laboratory Research And Exploration, 2015, 9:34-37.

[2] Li Zhonghua. Design of remote power meter reading system based on low voltage power line carrier [J]. Scientific and technical information, 2008,8:40-41.

[3] Xu Ying, Ma Weihua. Design of computer network meter reading system[J]. Automation technology and application, 2002,21 (3): 39-40.

[4] Li Jiahong. Research and development of school intelligent power management system[J]. technology vision, 2016, 6:25-27.

[5] Wang Di, high Qinxiang. Research on power grid friendly household electricity management optimization and control system [J]. Measurement and instrumentation, 2015, 9:18-20. 